\title{
BreastCare
}

\section{New Avenues in Secondary and Tertiary Prevention of Breast Cancer}

\author{
Rita K. Schmutzler \\ Center for Familial Breast and Ovarian Cancer, University Hospital of Cologne, Germany
}

While hereditary cancer subtypes were long considered of limited importance in the effort of defeating cancer, we now realize that they are of utmost importance in this battle. Based on recent achievements in deciphering the genetic background of breast and ovarian cancer we are facing a paradigmatic change in secondary and tertiary prevention from general screening to risk-adapted surveillance and from 'one size fits all' to targeted therapies. How come?

While all cancers arise as a result of somatically acquired mutations, hereditary tumors represent subtypes where the causative mutation is known. Given the tremendous amount of genetic aberrations found in a cancer genome this constitutes a huge advantage as the driver mutation that is causally implicated in oncogenesis can be distinguished from the vast number of passenger mutations that neither contribute to cancer development nor help in the search for targeted therapies. Meanwhile, we also learned that sporadic cancers can mimic their hereditary counterparts: they are initiated by somatic mutations in hereditary cancer genes and thus by definition belong to the same distinct tumor subtype. This has already been shown for $B R C A 1$ and BRCA2 and is likely to be true for further genes to be discovered. Somatic mutations in these genes may be responsible for the missing heritability that seems to confer a strong genetic heterogeneity. So how does this impact clinical management?

We already learned a lot from BRCA1/2-associated cancers in that they represent distinct tumor subtypes not only with respect to tumor initiation and disease penetrance but equally important with respect to genotype-/phenotype correlations, e.g. histological characteristics and amenability of preventive measures and targeted therapies. The articles in this focus are devoted to these aspects and emerging challenges: Engel and Fischer [1] outline the pitfalls of risk prediction and the necessity of prospective cohort studies for reliable risk calculations. Wittersheim et al. [2] illustrate strong genotype-/phenotype correlations in tumor histology that are important not only as pre- screening tools for hereditary cancer subtypes but also as prognostic and predictive biomarkers for the initiation of appropriate preventive and therapeutic measures. Consequently, Bick [3] argues for the importance of diagnostic studies in order to define appropriate screening modalities that need to be proven in long-term prospective studies to show a survival benefit. Finally, Kast and Rhiem [4] outline a straightforward strategy to target the Achilles heel of $B R C A$-associated tumors. This allowed for the first time to employ the concept of 'synthetic lethality' by using PARP inhibitors, a group of pharmacological inhibitors that just enter the clinical arena.

Besides the tremendous achievements in prediction, prevention, and treatment of hereditary breast and ovarian cancer these articles also outline how and what we can learn from hereditary tumor subtypes and how this can be translated into new concepts for riskadapted prevention and new treatment strategies for sporadic breast and ovarian cancer. They illustrate that hereditary cancer subtypes constitute prototypes for deciphering molecular oncogenesis and its targetability. In order to gain further scientific insights via this promising avenue there is one sine qua non, i.e. the establishment of large prospective cohort studies of hereditary subtypes. Such cohorts constitute enriched sample sets for the identification of genotype/phenotype correlations as a prerequisite for the introduction of new prevention concepts and the employment of new therapeutic regimens. This has recently become even more important since the missing heritability seems to be based on an extreme genetic heterogeneity transmitted by oligogenetic traits; a circumstance that encouraged efforts to accumulate national cohorts in international mega consortia. Although this constitutes a huge logistic challenge, every effort should be made to realize and consolidate these cohorts, as they are currently one of the most promising ways to gain further insights into risk prediction and drug targets relevant for more efficient screening concepts as well as new treatment options.

\section{References}

1 Engel C, Fischer C: Breast cancer risks and risk prediction models. Breast Care 2015;10: DOI:10.1159/000376600.

2 Wittersheim M, Büttner R, Markiefka B: Genotype/phenotype correlations in patients with hereditary breast cancer. Breast Care 2015;10: DOI:10.1159/000380900.
Bick U: Intensified surveillance for early detection of breast cancer in high-risk patients. Breast Care 2015;10: DOI:10.1159/000375390.
4 Kast K, Rhiem K: Familial breast cancer - targeted therapy in secondary and tertiary prevention. Breast Care 2015;10: DOI:10.1159/000380756.

\section{KARGER}

(C) 2015 S. Karger GmbH, Freiburg

$1661-3791 / 15 / 0101-0006 \$ 39.50 / 0$

Fax +497614520714
Univ. Prof. Dr. med. Rita Schmutzler

am Universitätsklinikum Köln (AöR)

Kerpener Str. 34, 50931 Köln

Rita.Schmutzler@uk-koeln.de 